\title{
The heat flow of Europa
}

\author{
Javier Ruiz* \\ Departamento de Geodinámica, Facultad de Ciencias Geológicas, Universidad Complutense de Madrid, 28040 Madrid, Spain
}

Received 5 July 2004; revised 22 November 2004

Available online 25 May 2005

\begin{abstract}
The heat flow from Europa has profound implications for ice shell thickness and structure, as well as for the existence of an internal ocean, which is strongly suggested by magnetic data. The brittle-ductile transition depth and the effective elastic thickness of the lithosphere are here used to perform heat flow estimations for Europa. Results give preferred heat flow values (for a typical geological strain rate of $10^{-15} \mathrm{~s}^{-1}$ ) of $70-110 \mathrm{~mW} \mathrm{~m}^{-2}$ for a brittle-ductile transition $2 \mathrm{~km}$ deep (the usually accepted upper limit for the brittle-ductile transition depth in the ice shell of Europa), $24-35 \mathrm{~mW} \mathrm{~m}^{-2}$ for an effective elastic thickness of $2.9 \mathrm{~km}$ supporting a plateau near the Cilix impact crater, and $>130 \mathrm{~mW} \mathrm{~m}^{-2}$ for effective elastic thicknesses of $\leqslant 0.4 \mathrm{~km}$ proposed for the lithosphere loaded by ridges and domes. These values are clearly higher than those produced by radiogenic heating, thus implying an important role for tidal heating. The $\geqslant 19-25 \mathrm{~km}$ thick ice shell proposed from the analysis of size and depth of impact structures suggests a heat flow of $\leqslant 30-45 \mathrm{~mW} \mathrm{~m}^{-2}$ reaching the ice shell base, which in turn would imply an important contribution to the heat flow from tidal heating within the ice shell. Tidally heated convection in the ice shell could be capable to supply $\sim 100 \mathrm{~mW} \mathrm{~m}^{-2}$ for superplastic flow, and, at the Cilix crater region, $\sim 35-50 \mathrm{~mW} \mathrm{~m}^{-2}$ for dislocation creep, which suggests local variations in the dominant flow mechanism for convection. The very high heat flows maybe related to ridges and domes could be originated by preferential heating at special settings.
\end{abstract}

Keywords: Europa; Satellites of Jupiter; Thermal histories; Tides, solid body

\section{Introduction}

Heat flow is a fundamental factor for understanding the evolution and present-day state of a planetary body, and so it is an important parameter to constraint geodynamic models. In Europa's case, the heat flow value has profound implications for ice shell thickness and structure, and for the possible existence of an internal ocean. It is therefore related (although not in a simple way) to the famous thin shell vs thick shell debate. The extreme positions in this debate defend, respectively, a shell thermally conductive a few kilometers thick (e.g., Greenberg et al., 2000, 2002), or a shell a few tens of kilometers thick and maybe convective in its lower part (e.g., Pappalardo et al., 1999; Pappalardo and Head, 2001). The analysis of size and depth of the largest

\footnotetext{
* Fax: +34 913944845.

E-mail address: jaruiz@geo.ucm.es.
}

impact structures suggest that these features were formed in an icy shell at least $\sim 19-25 \mathrm{~km}$ thick (Schenk, 2002), supporting a thick or (at least) relatively thick shell. Otherwise, there are solid evidences in favor of the existence of an internal ocean beneath the icy shell (Khurana et al., 1998; Kivelson et al., 2000), whose top would be $\sim 20 \mathrm{~km}$ below the surface if its electrical conductivity is similar to that of terrestrial sea water (Schilling et al., 2004).

Previous works have performed estimates of heat flows for Europa from the depth to the brittle-ductile transition and from the effective elastic thickness of the lithosphere. On the basis of the geological evidence it has been suggested that the depth to the brittle-ductile transition in the europan icy shell would be $2 \mathrm{~km}$ at the most (e.g., Pappalardo et al., 1999). The brittle-ductile transition marks the depth at which temperatures are high enough to permit ductile (and temperature-dependent) creep to be dominant over brittle failure as deformation mechanism. As creep is temperature-dependent, the depth to the brittle-ductile tran- 
sition can be used as a heat flow indicator: it has been proposed that heat flows of at least $\sim 100-200 \mathrm{~mW} \mathrm{~m}^{-2}$ are needed to put the brittle-ductile transition at a depth $\leqslant 2 \mathrm{~km}$ (Ruiz and Tejero, 1999, 2000; Pappalardo et al., 1999; McKinnon, 2000). Similarly, McKinnon et al. (2002) obtained a minimum heat flow of $75 \mathrm{~mW} \mathrm{~m}^{-2}$ from an extensional/compressional lithospheric instability model of folding at Astypalaea Linea. On the other hand, Nimmo et al. (2003) calculated the effective elastic thickness supporting a plateau in the Cilix crater region, deducing a heat flow of $34 \mathrm{~mW} \mathrm{~m}^{-2}$ (see Section 3), clearly lower than above mentioned values.

These heat flow values can be compared with results of theoretical models of tidal and radiogenic heat dissipation for Europa. Early models obtained heat flows $\leqslant 50 \mathrm{~mW} \mathrm{~m}^{-2}$ (Cassen et al., 1982; Squyres et al., 1983; Ross and Schubert, 1987; Ojakangas and Stevenson, 1989). Some estimates of tidal heating in the rocky portion of Europa, based on scalings of total dissipation in Io, have yielded heat flow values in the range $\sim 190-290 \mathrm{~mW} \mathrm{~m}^{-2}$ (Geissler et al., 2001; Thomson and Delaney, 2001; O'Brien et al., 2002), which would stabilize a conductive ice shell $\sim 2-3 \mathrm{~km}$ thick (clearly thinner than suggested from impact crater analyses). On the other hand, recent analyses (Hussmann et al., 2002; Nimmo and Manga, 2002; Ruiz and Tejero, 2003; Tobie et al., 2003; Showman and Han, 2004) of heat transfer in a Europa's convective ice shell have given heat flows in the range of $\sim 20-150 \mathrm{~mW} \mathrm{~m}^{-2}$.

In this paper I first realize a re-evaluation of heat flow estimations for Europa deduced from the brittle-ductile transition depth, in terms of strain rate. Then, I use effective elastic thicknesses in order to obtain independent estimations of heat flow. The relation between ice shell thickness and heat flow reaching the ice shell base, from the rock and metal core, is also discussed. The calculations are based solely on the physical properties of pure water ice. Substances such as ammonia (e.g., Cassen et al., 1982; Spohn and Schubert, 2003) or salts (e.g., Kargel et al., 2000; Prieto-Ballesteros and Kargel, 2005) may exist in the ice shell, but it is not known whether in sufficient amounts to significantly modify the rheological or thermal properties of water ice.

\section{Heat flow from the brittle-ductile transition depth}

For Europa, it is usual to put the brittle-ductile transition $2 \mathrm{~km}$ deep at most (e.g., Pappalardo et al., 1998, 1999; McKinnon, 2000). For example, the undulations found in Astypalaea Linea, interpreted as folds (Prockter and Pappalardo, 2000), have a wavelength of $\sim 25 \mathrm{~km}$, which would imply a brittle-ductile transition $\sim 2 \mathrm{~km}$ deep (McKinnon, 2000). Undulations with a similar spacing have also been observed in the leading hemisphere of Europa (Figueredo and Greeley, 2000). On the other hand, troughs, possibly grabens, in the Callanish and Tyre multiring impact struc-

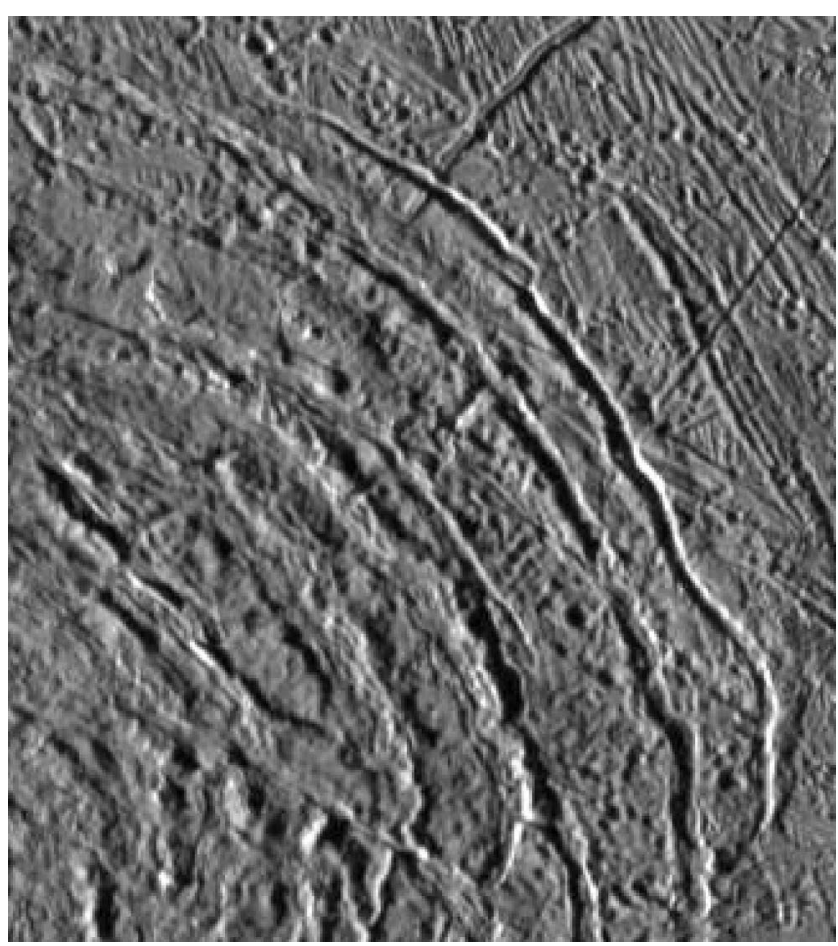

Fig. 1. Troughs northeast Tyre impact structure are seen in a mosaic of Galileo images taken during orbit E14. Troughs are up to $\sim 1.5-2 \mathrm{~km}$ wide, suggesting a brittle-ductile transition $\sim 1-2 \mathrm{~km}$ deep.

tures are up to $0.8-2 \mathrm{~km}$ wide (see Fig. 1): if these troughs are interpreted as grabens then its width implies a depth of faulting of $\sim 1-2 \mathrm{~km}$, which could correspond to the brittleductile transition (Moore et al., 1998; McKinnon, 2000).

Previous heat flow calculations by Ruiz and Tejero $(1999,2000)$ and Pappalardo et al. (1999) used a strain rate of $2 \times 10^{-10} \mathrm{~s}^{-1}$, which is roughly the mean value for tidally induced strain rates in a floating ice shell on Europa (Ojakangas and Stevenson, 1989), but geological processes on Europa should involve slower strain rates, although relevant strain rates are unknown. Theoretical models of band opening suggest strain rates in the range $\sim 10^{-12}-10^{-15} \mathrm{~s}^{-1}$ (Nimmo, 2004a; Stempel et al., 2004). Non-synchronous rotation, which is apparently related to the origin and orientation of many features on Europa (e.g., Greenberg et al., 1998), would have associated strain rates of $\leqslant 10^{-14} \mathrm{~s}^{-1}$ (Nimmo et al., 2003; Manga and Sinton, 2004). Thus, here I recalculate the heat flow corresponding to a brittle-ductile transition $2 \mathrm{~km}$ deep in terms of the strain rate. I consider results for $\sim 10^{-15} \mathrm{~s}^{-1}$ as most representatives, since this value is typical for many geological processes; results for tidal strain rates are also of interest, because they provide solid upper limits to the calculations.

Assuming preexisting planes of fractures of all orientations (in the absence of pore fluid pressure, as expected for an icy satellite), the brittle strength in the icy shell is given, for stress regime of compression and tension respectively, by (see Ruiz and Tejero, 2000)

$\left(\sigma_{1}-\sigma_{3}\right)_{\text {comp }}=2(\mu \rho g z+S) B$ 
$\left(\sigma_{1}-\sigma_{3}\right)_{\mathrm{ten}}=\frac{2(\mu \rho g z+S) B}{2 \mu B+1}$,

where $\mu$ is the friction coefficient, $z$ is the depth, $\rho$ is the density, $g$ is the gravity, $S$ is the material's cohesion, and $B=\left(\mu^{2}+1\right)^{1 / 2}+\mu$; for water ice $\mu=0.55$ and $S=1 \mathrm{MPa}$ (Beeman et al., 1988), and $\rho=930 \mathrm{~kg} \mathrm{~m}^{-3}$; for Europa $g=$ $1.31 \mathrm{~m} \mathrm{~s}^{-2}$.

In turn, the ductile strength of water ice is given by

$\left(\sigma_{1}-\sigma_{3}\right)_{\mathrm{d}}=\left(\frac{\dot{\varepsilon} d^{p}}{A}\right)^{1 / n} \exp \left(\frac{Q}{n R T}\right)$,

where $\dot{\varepsilon}$ is the strain rate, $A, p$, and $n$ are laboratorydetermined constants, $d$ is the grain size, $Q$ is the activation energy of creep, $R=8.3145 \mathrm{~J} \mathrm{~mol}^{-1} \mathrm{~K}^{-1}$ is the gas constant, and $T$ is the absolute temperature. In planetary conditions, water ice creep mainly should occur by superplastic (grain boundary sliding dominated) flow, which is grain size-sensitive, or dislocation creep, which is independent of grain size (for reviews of water ice rheology see Durham and Stern, 2001; Goldsby and Kohlstedt, 2001). For superplastic flow at $T<255 \mathrm{~K}$ (the relevant case here) $Q=49 \mathrm{~kJ} \mathrm{~mol}^{-1}, A=3.9 \times 10^{-3} \mathrm{MPa}^{-n} \mathrm{~m}^{p} \mathrm{~s}^{-1}, p=1.4$, and $n=1.8$ (Goldsby and Kohlstedt, 2001); for dislocation at $T<240 \mathrm{~K}$ creep $Q=61 \mathrm{~kJ} \mathrm{~mol}^{-1}, A=1.26 \times$ $10^{5} \mathrm{MPa}^{-n} \mathrm{~s}^{-1}, p=0$ and $n=4$ (e.g., Durham and Stern, 2001).

Superplastic flow is considered by some authors as the prevailing ductile deformation mechanism on Europa (Pappalardo et al., 1998; McKinnon, 1999; Goldsby and Kohlstedt, 2001), although high differential stresses (and differential stresses of at least several megapascals are needed to equal the brittle strength at the brittle-ductile transition (Ruiz and Tejero, 2000)), warm temperatures and ice crystal growth (which is in turn temperature-enhanced) increase the contribution of dislocation creep. It has therefore been suggested that this mechanism should also be taken into account for Europa's ice shell modeling (Durham et al., 2001). Thus, the calculations have been performed for both superplastic flow and dislocation creep, although this kind of calculation is relatively insensitive to the water ice rheology, as evidenced by previous works (Ruiz and Tejero, 2000; Ruiz, 2003). Convective processes are much more rheologysensitive than lithospheric processes, but they are beyond the scope of this paper.

For superplastic flow, grain sizes of $d=0.1$ and $1 \mathrm{~mm}$ were used in the calculations. Spectral analysis of a young fracture in the Tyre region suggests that the mean grain size of the shallow subsurface layer is $0.1 \mathrm{~mm}$ or greater (Geissler et al., 1998). Although this local and shallow value may not be relevant for geodynamics calculations, a grain size lower than $0.1 \mathrm{~mm}$ would not be reasonable if there are no impurities, which limit the crystal growth (McKinnon, 1999). On the other hand, dislocation creep becomes an important flow mechanism when grain sizes are bigger than $\sim 1 \mathrm{~mm}$ (McKinnon, 1999; Durham et al., 2001).

The knowledge of the depth to the temperature $T_{z}$ allows the calculation of the vertical heat flow. Although tidal heating in the ice shell must importantly contribute to the Europa's heat budget (e.g., Cassen et al., 1982; Squyres et al., 1983; Ross and Schubert, 1987; Ojakangas and Stevenson, 1989), this effect is strongly temperature-dependent (Ojakangas and Stevenson, 1989), and the part of the shell that contributes most to total heat flow is the warm, deep, and maybe convective ice near its base. On these grounds, it may be considered that the ice lithosphere (the outer and cold layer which may support geological stresses) is heated from below. So, taking a temperature-dependent thermal conductivity for water ice according to $k=k_{0} / T$, the heat flow is given by

$F=\frac{k_{0}}{z} \ln \left(\frac{T_{z}}{T_{\mathrm{S}}}\right)$,

where $k_{0}=567 \mathrm{~W} \mathrm{~m}^{-1}$ (Klinger, 1980), and $T_{\mathrm{S}}$ is the surface temperature.

The temperature at the brittle-ductile transition depth is obtained by equating Eqs. (1a) or (1b) and (2) for $z=z_{\text {BDT }}$. The surface temperature is here taken as $100 \mathrm{~K}$, which is considered as representative of the mean temperature at Europa's surface (e.g., Ojakangas and Stevenson, 1989).

The results for $z_{\mathrm{BDT}}=2 \mathrm{~km}$ are shown in Fig. 2, and it can be seen that heat flow values for superplastic flow and dislocation creep are similar. Complementarily, Fig. 3 shows heat flows for dislocation creep and strain rates of $10^{-15} \mathrm{~s}^{-1}$ in terms of brittle-ductile transition depth; heat flows for strain rates of $2 \times 10^{-10} \mathrm{~s}^{-1}$ in terms of brittle-ductile transition depth were obtained by Ruiz and Tejero (2000). For $z_{\mathrm{BDT}}=2$, a typical geological strain rate of $10^{-15} \mathrm{~s}^{-1} \mathrm{im}$ plies a heat flow of $70-110 \mathrm{~mW} \mathrm{~m}^{-2}$, and a tidal strain of $2 \times 10^{-10} \mathrm{~s}^{-1}$ puts an upper limit of $160-210 \mathrm{~mW} \mathrm{~m}^{-2}$. Summarizing, a brittle-ductile transition $2 \mathrm{~km}$ deep is consistent with heat flows of $70-210 \mathrm{~mW} \mathrm{~m}^{-2}$; values toward the lowermost part of this range are probably more representatives.

\section{Heat flow from effective elastic thicknesses}

The methodology described by McNutt (1984), which relates effective elastic thickness, curvature of an elastic plate and the strength envelope of the lithosphere, also permit to compute heat flows. This methodology is based in that the bending moment of the mechanical lithosphere must be the same that the bending moment of the equivalent elastic plate. The bending moment of the elastic plate is

$M=\frac{E K T_{\mathrm{e}}^{3}}{12\left(1-v^{2}\right)}$,

where $E$ is the Young's modulus, $K$ is the topography curvature, $T_{\mathrm{e}}$ is the effective elastic thickness, and $v$ is the Pois- 


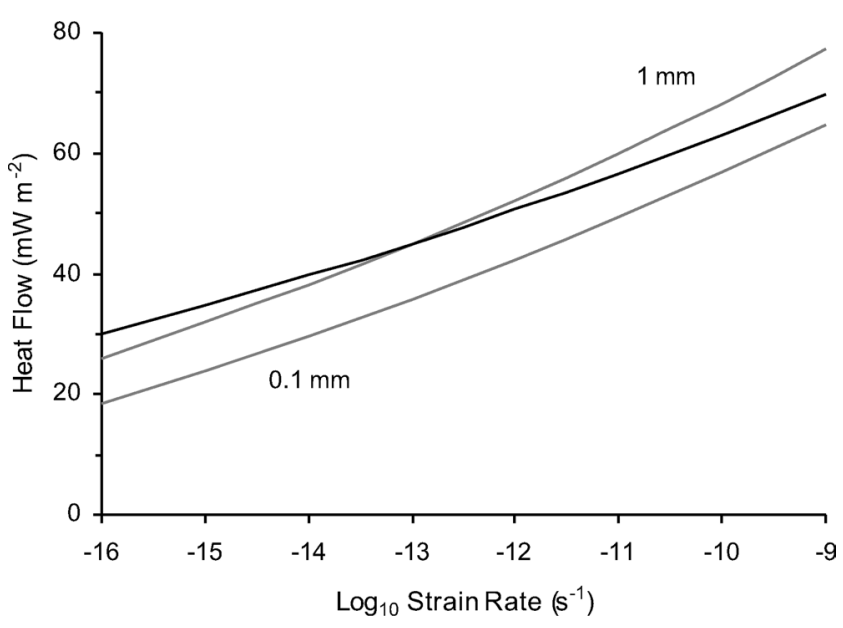

Fig. 4. Heat flow deduced from effective elastic thickness of $2.9 \mathrm{~km}$ for the lithosphere supporting a plateau southwest of Cilix impact crater. Surface temperature is taken as $110 \mathrm{~K}$, according to the location of this feature close to the equator. Black curve represents ductile deformation due to dislocation creep. Gray curves indicate ductile deformation due to superplastic (grain boundary sliding dominated) flow for grain sizes of 0.1 and $1 \mathrm{~mm}$.

I use the effective elastic thickness of $2.9 \mathrm{~km}$ and a maximum topography curvature of $7.5 \times 10^{-7} \mathrm{~m}^{-1}$ (Nimmo, $2004 b$ ), in order to perform a more accurate heat flow calculation from the equivalent strength envelope methodology, in terms of strain rate and by using superplastic flow (with grain sizes of 0.1 and $1 \mathrm{~mm}$ ) and dislocation creep as the dominant creep mechanism. Since this region is located close to the equator I use a surface temperature of $110 \mathrm{~K}$ (Ojakangas and Stevenson, 1989).

Results are shown in Fig. 4. For a strain rate of $10^{-15} \mathrm{~s}^{-1}$ the heat flow is $24-35 \mathrm{~mW} \mathrm{~m}^{-2}$, and an upper limits of $57-69 \mathrm{~mW} \mathrm{~m}^{-2}$ can be obtained from a tidal strain rate of $1.2 \times 10^{-10} \mathrm{~s}^{-1}$ (appropriate for this region; see Fig. 1 in Ojakangas and Stevenson, 1989). So, $\sim 25-70 \mathrm{~mW} \mathrm{~m}^{-2}$ is a permissive range for the heat flow deduced from this feature, with values in the lower part of this range being maybe more realistic. Effective elastic thickness values of 2.9 and $6 \mathrm{~km}$ are apparently very different. As stated above, the effective elastic thickness is a measure of lithospheric strength, and not a real layer thickness. The calculation of the effective elastic thickness is dependent on the used Young modulus value (the lower the Young modulus the higher the effective elastic thickness), and the use of different set of $T_{\mathrm{e}}$ and $E$ values does not necessarily changes the obtained heat flows in a substantial manner, although I prefer, by consistency with rheological laws experimentally obtained, to use the laboratory measure for Young modulus. For example, for $E=1 \mathrm{GPa}, T_{\mathrm{e}}=6 \mathrm{~km}$, the heat flow is $21-32 \mathrm{~mW} \mathrm{~m}^{-2}$ for $\dot{\varepsilon}=10^{-15} \mathrm{~s}^{-1}$, and $50-60 \mathrm{~mW} \mathrm{~m}^{-2}$ for $\dot{\varepsilon}=1.2 \times 10^{-10} \mathrm{~s}^{-1}$.

Europan ridges and domes could also load the icy shell. Observation of loading effects could be used to calculate the effective elastic thickness of the lithosphere. On the basis of photoclinometric profiles, Hurford et al. (2004) have proposed the existence of flexural bulges flanking ridges, and then have used distances between bulges' crests and ridges

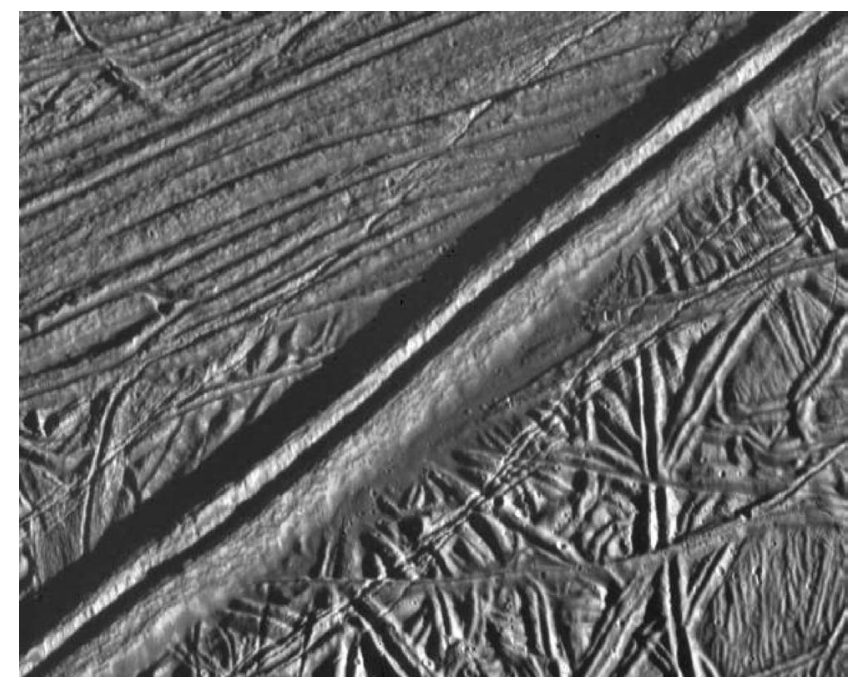

Fig. 5. The remarkable double ridge Androgeos Linea is flanked by fractures, separated roughly $3 \mathrm{~km}$ from axis ridge. Fractures were probably caused by ridge load.

axis to calculate (for $E=9 \mathrm{GPa}$ ) effective elastic thicknesses lower (with a unique exception) than $400 \mathrm{~m}$. On the other hand, Billings and Kattenhorn (2002) used distance to cracks flanking three ridges (Fig. 5) as indicators of the maximum tensile stress position in the flexed layer, and then this distance was utilized to calculate elastic thicknesses of 0.2 $2.6 \mathrm{~km}$, depending on the feature and parameter values used. Similarly, Williams and Greeley (1998) deduced an elastic thickness of $0.15-1.0 \mathrm{~km}$ from the bulge caused by a dome surrounding Conamara Chaos.

Works by Williams and Greeley (1998) and Billings and Kattenhorn (2002) used a density of $1186 \mathrm{~kg} \mathrm{~m}^{-3}$, appropriated for the eutectic liquid of the ternary system $\mathrm{MgSO}_{4}-$ $\mathrm{Na}_{2} \mathrm{SO}_{4}-\mathrm{H}_{2} \mathrm{O}$, for the material beneath the elastic layer. Using the density of a liquid for the material underlying the elastic layer is not an adequate procedure, since, as stated above, the effective elastic thickness is not the thickness of a real layer. Re-scaling their results for ice density and $E=9 \mathrm{GPa}$, effective elastic thicknesses of $\sim 0.1-0.4 \mathrm{~km}$ are obtained for the case of the fractures flanking ridges (which is consistent with the results in Hurford et al., 2004) and $\sim 0.1-0.2 \mathrm{~km}$ for the dome case.

These effective elastic thicknesses can also be converted to heat flow by using the procedure described here. For the case of effective elastic thicknesses $\leqslant 0.4 \mathrm{~km}$, and assuming topography curvatures as high as $10^{-5} \mathrm{~m}^{-1}$ in order to obtain lower limits (the curvature is taken concave downward, since maximum curvature is located in the flexural bulge for layers flexed by ridges and domes; see Turcotte and Schubert, 2002), the heat flow should be higher than $\sim 130$ 170 for a strain rate of $10^{-15} \mathrm{~s}^{-1}$. These values are higher than those calculated in the previous section, and probably represent very local conditions. Ridges and domes could nevertheless have been formed in places of amplified heating, by shear heating at fractures (Gaidos and Nimmo, 2000; 
Nimmo and Gaidos, 2002) or by tidal heating at raising warm diapirs (McKinnon, 1999; Sotin et al., 2002). In this case, elastic thicknesses proposed from ridges and domes would not be representative of the Europa lithosphere, and so the implied heat flows are probably biased.

Finally, Figueredo et al. (2002) proposed an elastic thickness (for $E=9 \mathrm{MPa}$ ) of $\sim 4 \pm 2 \mathrm{~km}$ for Murias Chaos region. This range is very wide and cannot be used to obtain precise heat flow estimations.

\section{The heat flow reaching the ice shell base}

If the whole ice shell is thermally conductive and has not internal heat sources (i.e., the shell is heated from below), the relation between heat flow and shell thickness is determined by ice thermal conductivity and surface temperature. The temperature at the base of the ice shell is given by the ice melting point, which is pressure-dependent according to (Chizhov, 1993)

$T_{\text {melting }}=273.16\left(1-\frac{P}{395.2 \mathrm{MPa}}\right)^{1 / 9}$,

where $P$ is pressure. Taking $P=\rho g z$ and $z=$ (total ice thickness), Eq. (9) can be inserted in Eq. (3) in order to calculate the heat flow corresponding to a given shell thickness. Fig. 6 shows results for $T_{\mathrm{s}}=100 \mathrm{~K}$. For a conductive shell at least $\sim 19-25 \mathrm{~km}$ thick (Schenk, 2002) the heat flow should be at most $\sim 20-30 \mathrm{~mW} \mathrm{~m}^{-2}$. As previously mentioned, a certain contribution to the total heat flow of Europa should come from tidal heating within the icy shell. So, these heat flow values must be considered as upper limits to the heat flow reaching the icy shell base from the rock and metal core (there is not significant tidal heating in an internal ocean).

But there is not necessarily a direct relation between heat flow and ice shell thickness. Indeed, the lower part of a thick (or relatively thick) europan ice shell can be convective (e.g.,

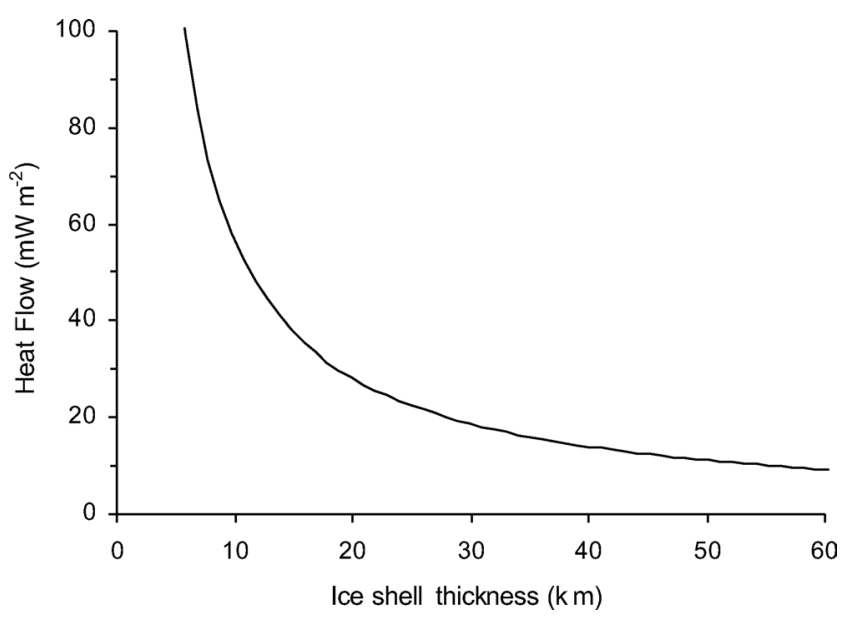

Fig. 6. Heat flow in terms of the thickness of a conductive ice shell on Europa. The temperature at the base of the shell is given by the ice melting point, which is pressure-dependent.
Pappalardo et al., 1998; McKinnon, 1999; Hussmann et al., 2002; Nimmo and Manga, 2002; Spohn and Schubert, 2003; Ruiz and Tejero, 2003; Tobie et al., 2003). In this case the relation between heat flow and shell thickness is more complicated than in the conductive one, and the heat flow reaching the ice shell base is not determined from observation of geological structures, which only inform about the conductive heat flow through the stagnant lid of the convective system. The conductive heat transfer in the stagnant lid is due to the heat flow from the actively convective sublayer, which can be tidally heated. Also, there can be a contribution from tidal heating in the warm convective ice. In any case, the analysis of stability against convection of a floating ice shell on Europa implies that the onset of convection requires conductive heat flow decreasing under $10-45 \mathrm{~mW} \mathrm{~m}^{-2}$ (Ruiz and Tejero, 2003), which are equivalent to a conductive ice shell at least $\sim 10-50 \mathrm{~km}$ thick, depending on the dominant flow mechanism.

Thus, an ice shell at least $\sim 20 \mathrm{~km}$ thick implies a heat flow of at most $\sim 30-45 \mathrm{~mW} \mathrm{~m}^{-2}$ reaching the shell base.

\section{Discussion}

Whereas there is a plenty of theoretical models of heat transfer on Europa (e.g., Cassen et al., 1982; Ojakangas and Stevenson, 1989; Hussmann et al., 2002; Nimmo and Manga, 2002; Barr and Pappalardo, 2003; Ruiz and Tejero, 2003; Spohn and Schubert, 2003; Tobie et al., 2003; Showman and Han, 2004), information on the true heat flow from this moon is very sparse.

From brittle-ductile transition depths or effective elastic thicknesses, reliable upper limits for the heat flow can be established by using tidal strain, but lower limits are much more difficult to obtain. The existence of a regolith surface layer (e.g., Ross and Schubert, 1987) or a solid-state greenhouse in the uppermost ice (e.g., Matson and Brown, 1989), could result in a significantly higher temperature very close to the surface, which is equivalent to an effective surface temperature higher than the observed, and to lowering the heat flow (Ruiz, 2003). Similarly, the presence of porosity would result in a reduction of the thermal conductivity, and consequently in a decrease in the calculated heat flow. The likelihood of porosity increases with proximity to the surface, which reinforces the possible reduction of the thermal conductivity, since cold temperatures predict a high thermal conductivity for pure (crystalline) ice. It is important to be aware of these possible effects, although they were not taken into account in the calculations here presented, due to that the extent of its influence is unknown.

Besides those complexities, the majority of indications on heat flows suggest high values when compared with that produced from present-day radiogenic heating (roughly applicable given the youth of europan surface, $\sim 30$ to $70 \mathrm{Myr}$ in average; Zahnle et al., 2003), which must contribute with $\sim 6-8 \mathrm{~mW} \mathrm{~m}^{-2}$ (Cassen et al., 1982; Squyres et al., 1983; 
Hussmann et al., 2002; Spohn and Schubert, 2003). This implies an important role for tidal heating on the dynamics of this satellite, as was proposed in classical works (e.g., Cassen et al., 1979, 1982; Squyres et al., 1983; Ross and Schubert, 1987; Ojakangas and Stevenson, 1989). Moreover, heat flow values calculated in Sections 2 and 3 are higher or similar respectively to the upper limits for the heat flow at the ice shell base discussed in Section 4, which implies an important (maybe dominant) contribution to the total Europa's heat budget from tidal heating within the ice shell.

Tidal heating in the ice shell could mainly occur in the warm interior of a convective layer (McKinnon, 1999; Ruiz and Tejero, 2000, 2003; McKinnon and Shock, 2001). Ruiz and Tejero (2003) have calculated equilibrium heat flows that can be generated and transferred (toward the stagnant lid base) by a convective ice layer heated from within by tidal dissipation. Although a certain amount of heat must enter the ice shell from below, convection heated from within can be taken as a valid approximation if the dominant contribution to the heat flow arises from tidal dissipation in the convective layer. These authors followed McKinnon (1999), which regards the use of Newtonian viscosity sufficient for convection if an average effective viscosity is appropriately defined in terms of tidal strain rates (taken as $2 \times 10^{-10} \mathrm{~s}^{-1}$ ), since tidal stresses on Europa are much higher that those due to thermal buoyancy. In this point, it is worth to make clear again that geological processes in the non-convective stagnant lid would be related to slower strain rates: a typical geological strain rate of $\sim 10^{-15} \mathrm{~s}^{-1}$ can be roughly appropriate for heat flow calculation based on the brittle-ductile transition depth or on the effective elastic thickness of the lithosphere.

Results in Ruiz and Tejero (2003) for superplastic flow are $80-130 \mathrm{~mW} \mathrm{~m}^{-2}$ (and an ice shell $\sim 15-50 \mathrm{~km}$ thick, including the essentially non-dissipative stagnant lid) for grain sizes of $0.1-1 \mathrm{~mm}$, values similar to the most likely range of $70-110 \mathrm{~mW} \mathrm{~m}^{-2}$ obtained for a brittle-ductile transition depth of $2 \mathrm{~km}$ (although grain size dependence have opposed tendencies in both set of calculations: increasing grain size decrease convective heat flow), and to the $>75 \mathrm{~mW} \mathrm{~m}^{-2}$ obtained by McKinnon et al. (2002) for folding at Astypalaea Linea. So, tidally heated convection in the ice shell with superplastic flow as dominant flow mechanism could be capable to explain heat flows of $\sim 100 \mathrm{~mW} \mathrm{~m}^{-2}$, in accordance with some geological observations. In this context very high heat flows possibly associated with fractures and domes would be originated by preferential heating at special settings.

On the other hand, Ruiz and Tejero (2003) obtained $\sim 40-$ $60 \mathrm{~mW} \mathrm{~m}^{-2}$ for dislocation creep. (According to Durham et al. (1997) two regimes characterize the flow law of dislocation creep for $T<258 \mathrm{~K}$, separated by a temperature of $240 \mathrm{~K}$, whereas Goldsby and Kohlstedt (2001) found one regime only, similar to the low temperature regime in Durham et al. (1997): both possibilities were considered in the calculations.) These values are closer to the preferred range of $24-35 \mathrm{~mW} \mathrm{~m}^{-2}$ calculated (from the effective elastic thickness) for the Cilix region than those obtained for superplastic flow. Moreover, if the methodology of these authors is applied by using $T_{\mathrm{s}}=110 \mathrm{~K}$ (in these calculations heat flow is almost independent of the surface temperature, but it is not the case for stagnant lid and whole shell thicknesses) and a tidal strain rate of $1.2 \times 10^{-10} \mathrm{~s}^{-1}$, the convective heat flow is $\sim 35-50 \mathrm{~mW} \mathrm{~m}^{-2}$ (and the shell thickness $\sim 30-40 \mathrm{~km}$ thick) for dislocation creep. This is consistent (still more if it is taken into account uncertainty in geological strain rates) with local variations in the dominant flow mechanism for convection, which in turn could arise from variations in the grain size (see Section 2). In any case, the main flow mechanism in the convective layer is not necessarily the same that the one dominating in the mechanical lithosphere.

Some authors have proposed that the geological record of Europa contains evidence of a shell thickening with time (e.g., Prockter et al., 1999; Pappalardo et al., 1999; Figueredo and Greeley, 2004), which could lead to the onset of convection from an initial entirely conductive shell. This inference is based on a proposed change in the style of resurfacing from mainly tectonic to destruction of preexistent terrains by chaos and lenticulae formation. Further work in many lines, including the precise temporal relation between features utilized as heat flow indicators, is needed in order to help to refine the thermal history. In any case, a tidally heated convective ice shell can satisfy the current constraints for heat flows and shell thickness on Europa.

\section{Acknowledgments}

I thank Francis Nimmo for discussion about some aspects of this work, David Stevenson and William Moore for reviews, and Francisco Anguita for help with the manuscript. The research was supported by a grant of the Spanish Secretaría de Estado de Educación y Universidades.

\section{References}

Barr, A.C., Pappalardo, R.T., 2003. Numerical simulations of nonNewtonian convection in ice: Application to Europa. Proc. Lunar Sci. Conf. 34. Abstract 1806.

Beeman, M., Durham, W.B., Kirby, S.H., 1988. Friction of ice. J. Geophys. Res. 93, 7625-7633.

Billings, S.E., Kattenhorn, S.A., 2002. Determination of ice crust thickness from franking cracks along ridges on Europa. Proc. Lunar Sci. Conf. 33. Abstract 1813

Cassen, P.M., Reynolds, R.T., Peale, S.J., 1979. Is there liquid water on Europa? Geophys. Res. Lett. 6, 731-734.

Cassen, P.M., Peale, S.J., Reynolds, R.T., 1982. Structure and thermal evolution of the Galilean satellites. In: Morrison, D. (Ed.), Satellites of Jupiter. Univ. of Arizona Press, Tucson, pp. 93-128.

Chizhov, V.E., 1993. Thermodynamic properties and thermal equation of state of high-pressure ice phases. Prikl. Mekh. Tekh. Fiz. 2, 113-123. (Engl. transl.) 
Durham, W.B., Stern, L.A., 2001. Rheological properties of water iceApplications to satellites of the outer planets. Annu. Rev. Earth Planet. Sci. 29, 295-330.

Durham, W.B., Kirby, S.H., Stern, L.A., 1997. Creep of water ices at planetary conditions: A compilation. J. Geophys. Res. 102 (16), 1629316302.

Durham, W.B., Stern, L.A., Kirby, S.H., 2001. Rheology of ice I at low stress and elevated confining pressure. J. Geophys. Res. 106, 1103111042.

Figueredo, P.H., Greeley, R., 2000. Geologic mapping of the northern hemisphere of Europa from Galileo solid-state imaging data. J. Geophys. Res. 105, 22629-22646.

Figueredo, P.H., Greeley, R., 2004. Resurfacic history of Europa from poleto-pole geologic mapping. Icarus 167, 287-312.

Figueredo, P.H., Chuang, F.C., Rathbun, J., Kirk, R.L., Greeley, R., 2002. Geology and origin of Europa's "Mitten" feature (Murias Chaos). J. Geophys. Res. 107, 10.1029/2001JE001591.

Gaidos, E.J., Nimmo, F., 2000. Tectonics and water on Europa. Nature 405 , 637.

Geissler, P.E., 16 colleagues, 1998. Evolution of lineaments on Europa: Clues from Galileo multispectral imaging observations. Icarus 135 , 107-126.

Geissler, P.E., O'Brien, D.P., Greenberg, R., 2001. Silicate volcanism on Europa. Proc. Lunar Sci. Conf. 32. Abstract 2068.

Goldsby, D.L., Kohlstedt, D.L., 2001. Superplastic deformation of ice: Experimental observations. J. Geophys. Res. 106, 11017-11030.

Greenberg, R., Geissler, P., Hoppa, G.V., Tufts, B.R., Durda, D.D., Pappalardo, R., Head, J.W., Greeley, R., Sullivan, R., Carr, M.H., 1998. Tectonic processes on Europa: Tidal stresses, mechanical response, and visible features. Icarus 135, 64-78.

Greenberg, R., Geissler, P., Tufts, B.R., Hoppa, G.V., 2000. Habitability of Europa's crust: The role of tidal-tectonic processes. J. Geophys. Res. 105, 17551-17562.

Greenberg, R., Geissler, P., Hoppa, G., Tufts, B.R., 2002. Tidal-tectonic processes and their implications for the character of the Europa's icy crust. Rev. Geophys. 40, 1004, 10.1029/2000RG000096.

Hurford, T.A., Preblich, B., Beyer, R.A., Greenberg, R., 2004. Flexure of Europa's lithosphere due to ridge loading. Proc. Lunar Sci. Conf. 35. Abstract 1831.

Hussmann, H., Spohn, T., Wieczerkowski, K., 2002. Thermal equilibrium states of Europa's ice shell: Implications for internal ocean thickness and heat flow. Icarus 156, 143-151.

Kargel, J.S., Kaye, J.Z., Head, J.W., Marion, G.M., Sassen, R., Crowley, J.K., Ballesteros, O.P., Grant, S.A., Hogenboom, D.L., 2000. Europa's crust and ocean: Origin, composition, and the prospects for life. Icarus 148, 226-265.

Khurana, K.K., Kivelson, M.G., Stevenson, D.J., Schubert, G., Russell, C.T., Walker, R.J., Polanskey, C., 1998. Induced magnetic fields as evidence for subsurface oceans in Europa and Callisto. Nature 395, 777-780.

Kivelson, M.G., Khurana, K.K., Russell, C.T., Volwerk, M., Walker, R.J., Zimmer, C., 2000. Galileo magnetometer measurements: A stronger case for a subsurface ocean at Europa. Science 289, 1340-1343.

Klinger, J., 1980. Influence of a phase transition of the ice on the heat and mass balance of comets. Science 209, 271-272.

Manga, N., Sinton, A., 2004. Formation of bands and ridges on Europa by cyclic deformation: Insights from analogue wax experiments. J. Geophys. Res. 109, E09001, 10.1029/2000JE001476.

Matson, D.L., Brown, R.H., 1989. Solid-state greenhouses and their implications for icy satellites. Icarus 77, 67-81.

McKinnon, W.B., 1999. Convective instability in Europa's floating ice shell. Geophys. Res. Lett. 26, 951-954.

McKinnon W.B., 2000. Europan heat flow and crustal thickness estimates from fold wavelengths and impact ring graben widths. In: 32nd Annual Meeting of the DPS. Abstract 38.01.

McKinnon, W.B., Shock, E.L., 2001. Ocean karma: What goes around on Europa (or does it?). Proc. Lunar Sci. Conf. 32. Abstract 2181.
McKinnon, W.B., Schenk, P.M., Dombard, A.J., 2002. Estimates of Europa's heat flow and ice thickness: Convergence at last? In: Second Astrobiology Science Conference. Abstract with Program, p. 50, NASA Ames Research Center, Moffett Field.

McNutt, M.K., 1984. Lithospheric flexure and thermal anomalies. J. Geophys. Res. 89, 11180-11194.

Moore, J.M., 17 colleagues, 1998. Large impact features on Europa: Results of the Galileo Nominal Mission. Icarus 135, 127-145.

Nimmo, F., 2004a. Dynamics of rifting and modes of extension on icy satellites. J. Geophys. Res. 109, E01003, 10.1029/2001JE001591.

Nimmo F., 2004b. What is the Young's modulus of ice? In: Proc. Europa's Icy Shell Conf. Abstract 7005.

Nimmo, F., Gaidos, E., 2002. Strike-slip motion and double ridge formation on Europa. J. Geophys. Res. 107, 10.1029/2000JE001476.

Nimmo, F., Manga, N., 2002. Causes, characteristics and consequences of convective diapirism on Europa. Geophys. Res. Lett. 29, 2109, 10.1029/2002GL015754.

Nimmo, F., Giese, B., Pappalardo, R.T., 2003. Estimates of Europa's ice shell thickness from elastically-supported topography. Geophys. Res. Lett. 30, 1233, 10.1029/2002GL016660.

O’Brien, D.P., Geissler, P., Greenberg, R., 2002. A melt-through model for chaos formation on Europa. Icarus 156, 152-161.

Ojakangas, G.W., Stevenson, D.J., 1989. Thermal state of an ice shell on Europa. Icarus 81, 220-241.

Pappalardo, R.T., Head, J.W., 2001. The thick-shell model of Europa's geology: Implications for crustal processes. Proc. Lunar Sci. Conf. 32. Abstract 1866.

Pappalardo, R.T., 10 colleagues, 1998. Geological evidence for solid-state convection in Europa's ice shell. Nature 391, 365-368.

Pappalardo, R.T., 31 colleagues, 1999. Does Europa have a subsurface ocean? Evaluation of the geological evidence. J. Geophys. Res. 104, 24015-24055.

Petrenko, V.F., Whitworth, R., 1999. Physics of Ice. Oxford Univ. Press, New York.

Prieto-Ballesteros, O., Kargel, J.S., 2005. Thermal state and complex geology of a heterogeneous salty crust of Jupiter's satellite, Europa. Icarus 173, 212-221.

Prockter, L.M., Pappalardo, R.T., 2000. Folds on Europa: Implications for crustal cycling and accommodation of extension. Science 289, 941-943.

Prockter, L.M., Antman, A.M., Pappalardo, R.T., Head, J.W., Collins, G.C., 1999. Europa: Stratigraphy and geological history of the anti-jovian region from Galileo E14 solid-state imaging data. J. Geophys. Res. 104, 16531-16540.

Ross, M.N., Schubert, G., 1987. Tidal heating in an internal ocean model of Europa. Nature 325, 133-134.

Ruiz, J., 2003. Heat flow and depth to a possible internal ocean on Triton. Icarus 166, 436-439.

Ruiz, J., Tejero, R., 1999. Heat flow and brittle-ductile transition in the ice shell of Europa. Proc. Lunar Sci. Conf. 32. Abstract 1031.

Ruiz, J., Tejero, R., 2000. Heat flows through the ice lithosphere of Europa. J. Geophys. Res. 105, 23283-23289.

Ruiz, J., Tejero, R., 2003. Heat flow, lenticulae spacing, and possibility of convection in the ice shell of Europa. Icarus 162, 362-373.

Schenk, P.M., 2002. Thickness constraints on the icy shells of Galilean satellites from a comparison of crater shapes. Nature 417, 419-421.

Schilling, N., Khurana, K.K., Kivelson, M.G., 2004. Limits on an intrinsic dipole moment in Europa. J. Geophys. Res. 109, E05006, 10.1029/2003JE002166.

Showman, A.P., Han, L., 2004. Numerical simulations of convection in Europa's ice shell: Implications for surface features. J. Geophys. Res. 109, E01010, 10.1029/2003JE002103.

Sotin, C., Head, J.W., Tobie, G., 2002. Europa: Tidal heating of upwelling thermal plumes and the origin of lenticulae and chaos melting. Geophys. Res. Lett. 29, 10.1029/2001GL013844.

Spohn, T., Schubert, G., 2003. Oceans in the icy Galilean satellites? Icarus 161, 456-467.

Squyres, S.W., Reynolds, R.T., Cassen, P.M., Peale, S.J., 1983. Liquid water and active resurfacing on Europa. Nature 301, 225-226. 
Stempel, M.M., Barr, A.C., Pappalardo, R.T., 2004. Constraints on the opening rate of bands on Europa. In: Proc. Europa's Icy Shell Conf. Abstract 7027.

Thomson, R.E., Delaney, J.R., 2001. Evidence for a weakly stratified europan ocean sustained by seafloor heat flux. J. Geophys. Res. 106, 12355-12365.

Tobie, G., Choblet, G., Sotin, C., 2003. Tidally heated convection: Constraints on Europa's ice shell thickness. J. Geophys. Res. 108, 5124, 10.1029/2003JE002099.

Turcotte, D.L., Schubert, G., 2002. Geodynamics, second ed. Cambridge Univ. Press, Cambridge.
Vaughan, D.G., 1995. Tidal flexure at ice shell margins. J. Geophys. Res. 100, 6213-6224.

Watts, A.B., 2001. Isostasy and Flexure of the Lithosphere. Cambridge Univ. Press, Cambridge.

Watts, A.B., Burov, E.B., 2003. Lithospheric strength and its relation to the elastic and seismogenetic layer thickness. Earth Planet. Sci. Lett. 213, $113-131$.

Williams, K.K., Greeley, R., 1998. Estimates of ice thickness in the Conamara Chaos region of Europa. Geophys. Res. Lett. 25, 4273-4276.

Zahnle, K., Schenk, P., Levison, H., Dones, L., 2003. Cratering rates in the outer Solar System. Icarus 163, 263-289. 